\title{
CASO CLÍNICO \\ Divertículos de intestino delgado en relación con la hemorragia de tubo digestivo. Informe de casos y revisión de la literatura
}

Cirujano General

Enero-Junio 2015

Vol. 37, núms. 1-2 / p. 49-55

\author{
Small bowel diverticula associated with digestive tract bleeding. \\ $A$ report on cases and a review of the literature
}

\author{
José Manuel Correa Rovelo, * Amado de Jesús Athié Athié,* \\ Luis Pablo Alessio Robles Landa, ${ }^{*}$ Lucero Georgina Reyes Espejel, ${ }^{*}$ \\ Jacobo Martínez Zárate, * José Mancera Andrade*
}

\begin{abstract}
Palabras clave: Hemorragia de tubo digestivo, divertículos duodenales, divertículos yeyunales.

Key words: Gastrointestinal bleeding, duodenal diverticulum, jejunal diverticulum.
\end{abstract}

* Departamento de Cirugía General del Hospital Médica Sur, México, DF.

Recibido: 01/01/2015 Aceptado: 01/05/2015

\section{RESUMEN}

El divertículo duodenal fue descrito por Chomel en 1710. En 1951, Patterson y Bromberg describieron el primer caso de hemorragia de tubo digestivo alto por divertículo duodenal. La frecuencia es variable dependiendo de la edad del paciente y el método diagnóstico. Los estudios radiográficos de tubo digestivo diagnostican enfermedad diverticular del duodeno de 1 a $5 \%$, y en series de autopsia, la incidencia es de 15 a $20 \%$. La hemorragia yeyunal o ileal supone entre 5 y $30 \%$ de las hemorragias digestivas recurrentes. Nuestro objetivo es presentar dos casos de hemorragia digestiva recurrente en el que se utilizaron diversas herramientas para el diagnóstico y tratamiento.

\section{INTRODUCCIÓN}

$\mathrm{E}^{\prime}$ divertículo duodenal fue descrito por Chomel en 1710. ${ }^{1}$ En 1951, Patterson y Bromberg describieron el primer caso de hemorragia de tubo digestivo alto por divertículo duodenal. La frecuencia es variable dependiendo de la edad del paciente y el método diagnóstico. Los estudios radiográficos de tubo digestivo diagnostican enfermedad diverticular del duodeno en 1 a 5\%, y en series de autopsia, la frecuencia es de 15 a 20\%. ${ }^{2}$ Se pueden clasificar como congénitos o adquiridos, verdaderos o falsos, intraluminales o extraluminales, siendo sintomáticos el 10\% de los casos; los síntomas son inespecíficos, como dolor abdominal, náuseas, vómitos, melena, hematemesis e ictericia. Como patogenia, se ha postulado que son las

\section{ABSTRACT}

Duodenal diverticulum was described by Chomel in 1710, and in 1951 Patterson and Bromberg described the first case of upper gastrointestinal bleeding from duodenal diverticulum. The incidence is variable depending on the age of the patient and the diagnostic method. Radiography studies of the digestive tract diagnose from 1 to $5 \%$ diverticular disease of the duodenum, and in autopsy series, the incidence is 15 to 20\%. Jejunal or ileal hemorrhage accounts for between 5 and $30 \%$ of recurrent gastrointestinal bleeding. In this study, two cases are presented of recurrent gastrointestinal bleeding in which different tools were used for diagnosis and treatment. fuerzas de pulsión las que producen herniación de la mucosa y la submucosa a través de la pared intestinal, siendo el defecto de la pared el sitio de entrada de los vasos perforantes o la ampolla de Vater. ${ }^{3,4}$

La hemorragia digestiva de origen yeyunal o ileal, aunque infrecuente, representa de 5 a $30 \%$ de las hemorragias digestivas recurrentes. Puede acompañarse de gran morbilidad y, con frecuencia, su diagnóstico causal es difícil. Las causas más frecuentes de hemorragia digestiva con origen en yeyuno o íleon son las malformaciones arteriovenosas y los tumores de intestino delgado; ${ }^{1,2}$ en cuanto a los divertículos, podemos hablar del divertículo de Meckel, que es un divertículo congénito o verdadero, cuya pared contiene todas las capas, a diferencia de los divertículos yeyunales, que son en su mayoría 
falsos, situados en el borde mesentérico del yeyeuno; ${ }^{5-7}$ un sangrado por estos últimos es relativamente raro.

Presentamos dos casos de hemorragia digestiva recurrente en los que se realizaron diferentes estudios para su tratamiento y diagnóstico.

\section{CASO CLÍNICO 1: DIVERTÍCULO DUODENAL}

Varón de 61 años de edad, sedentario, con diabetes mellitus (DM) tipo 2 diagnosticada en 2006, controlada con hipoglucemiantes orales, e hipertrigliceridemia tratada con fibratos; tabaquismo y alcoholismo positivos (de una a cinco copas de whisky al día). Antecedentes quirúrgicos de apendicectomía abierta en 1981, drenaje de hematoma de pared abdominal por trauma contuso de abdomen cerrado en 1983, colecistectomía abierta en 1987, artroplastia de codo derecho en 1985, plastia inguinal bilateral en 2001, transfusionales en dos ocasiones. Antecedente de gastroenteritis infecciosa de cuatro días, previa a su ingreso, tratada con ciprofloxacino. El paciente ingresó en el Servicio de Urgencias por referir evacuaciones hematoquécicas, negando otra sintomatología. Datos relevantes de la exploración física: tendencia a la hipotensión arterial, peristalsis incrementada de predominio en hemiabdomen izquierdo, tacto rectal con huellas de restos hemáticos de características hematoquécicas. Las pruebas de laboratorio

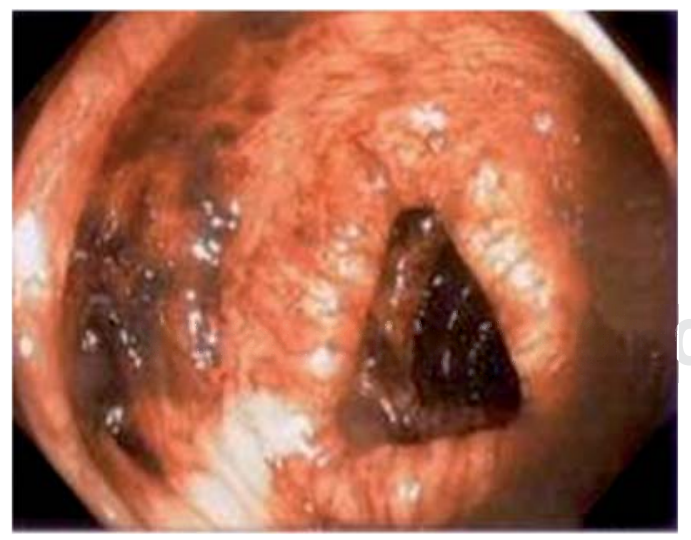

Figura 1. Colon transverso con datos compatibles de hemorragia de tubo digestivo. al ingreso reportaron una hemoglobina de $10.4 \mathrm{~g} / \mathrm{dL}$. Se realizó reposición de volumen y colocación de sonda nasogástrica con aspirado positivo para sangrado. Una vez estabilizado el paciente, se realizaron estudios endoscópicos: en la colonoscopia se evidenciaron múltiples divertículos en la región rectosigmoidea, sin huellas de sangrado reciente, logrando acceso hasta el ángulo hepático; la panendoscopia evidenció erosión de la mucosa a nivel de la unión esofagogástrica, gastritis leve y hemobilia a nivel de la segunda porción del duodeno, con imagen de divertículo con huellas de sangrado reciente (Figuras 1 y 2). Durante el procedimiento, el paciente desaturó hasta el $34 \%$, y ante el riesgo de resangrado y perforación, se decidió el ingreso a Unidad de Terapia Intensiva (UTI) para vigilancia estrecha (Figura 3); permaneció en dicha unidad durante 48 horas, con adecuada evolución. Se realizaron nuevamente estudios de laboratorio, con descenso de la hemoglobina a $7.4 \mathrm{~g} / \mathrm{dL}$, lo que requirió la transfusión de dos concentrados eritrocitarios. Fue egresado a piso, donde se inició tolerancia a la vía oral y se realizó una serie esófago-gastroduodenal, en la cual se evidenció divertículo duodenal en la segunda porción del duodeno, cara medial (Figura 4).

Se mantuvo en vigilancia, con evolución clínica favorable, y se decidió su egreso al cuarto día de estancia hospitalaria por mejoría significativa del cuadro de ingreso, sin datos de sangrado o perforación.

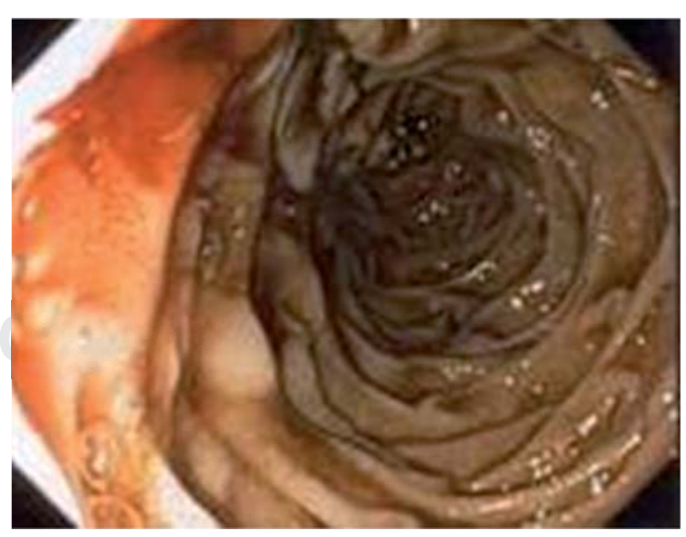

Figura 2. Segunda porción del duodeno, cara medial, imagen con hemorragia divertículo-duodenal. 


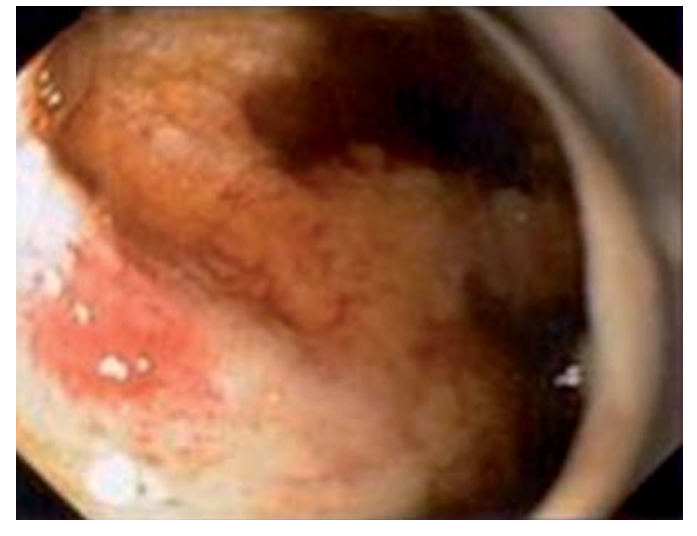

Figura 3. Endoscopio dentro del divertículo duodenal con desfacelamiento de la mucosa diverticular.

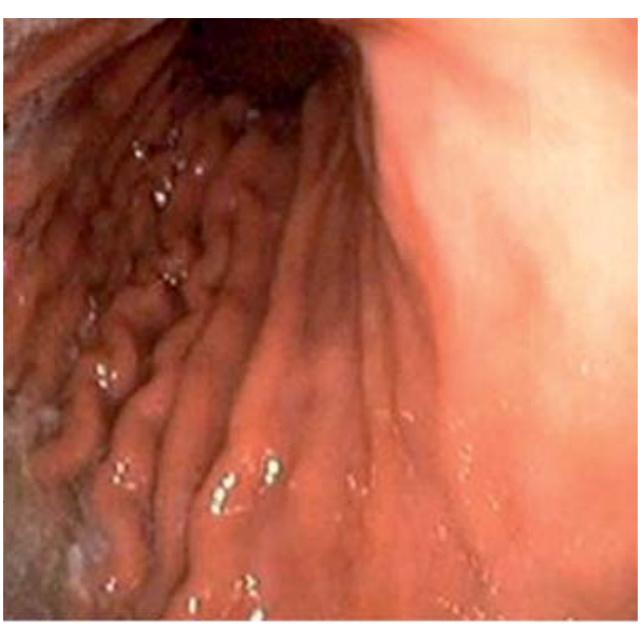

Figura 5. Endoscopia alta normal.

\section{CASO CLÍNICO 2: DIVERTÍCULOS EN YEYUNO}

Varón de 41 años, con cuadro clínico de cuatro días de evolución caracterizado por dolor abdominal difuso tipo cólico, escala visual análoga (EVA) 5/10, no irradiado, que mejoró con el reposo, acompañado de evacuaciones melénicas en número de cuatro al día. Al segundo día se agregó malestar generalizado con astenia, hiporexia, palidez generalizada de tegumentos y pérdida del estado de alerta, motivo por el cual acudió a un hospital no gubernamental en su lugar

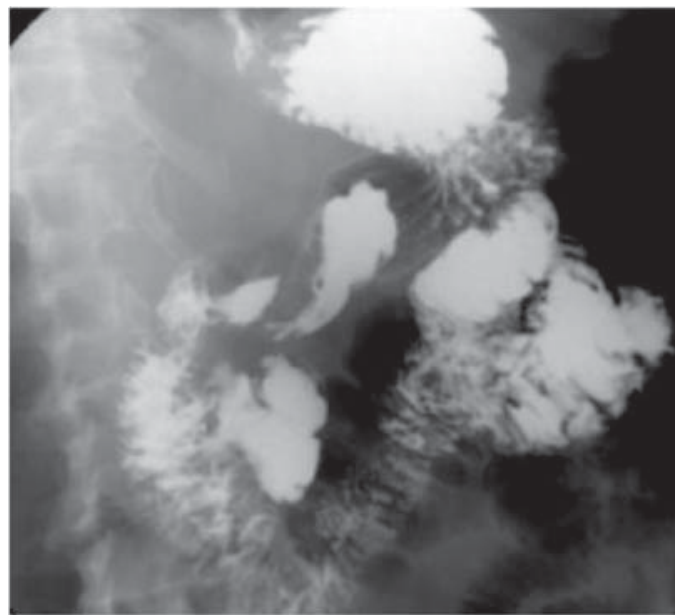

Figura 4. Serie esófago-gastroduodenal. Divertículo cara medial, segunda porción duodenal.

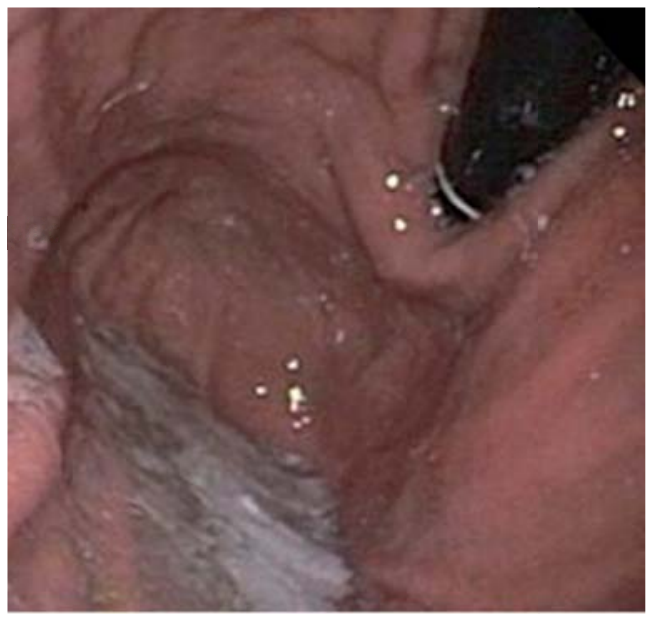

de origen, donde se le realizó una panendoscopia, la cual fue reportada como normal (Figura 5), pero durante su hospitalización persistió con las evacuaciones melénicas y disminución de la hemoglobina hasta $6.1 \mathrm{~g} /$ $\mathrm{dL}$, por lo cual se realizó hemotransfusión de tres paquetes globulares y colonoscopia con reporte de restos hemáticos, sin encontrar el sitio de sangrado (Figura 6). Se solicitó alta y referencia a nuestro hospital para continuar con el abordaje diagnóstico. Al ingreso en Urgencias, el paciente estaba alerta, con palidez generalizada de tegumentos y hemodinámicamente estable; se decidió su 

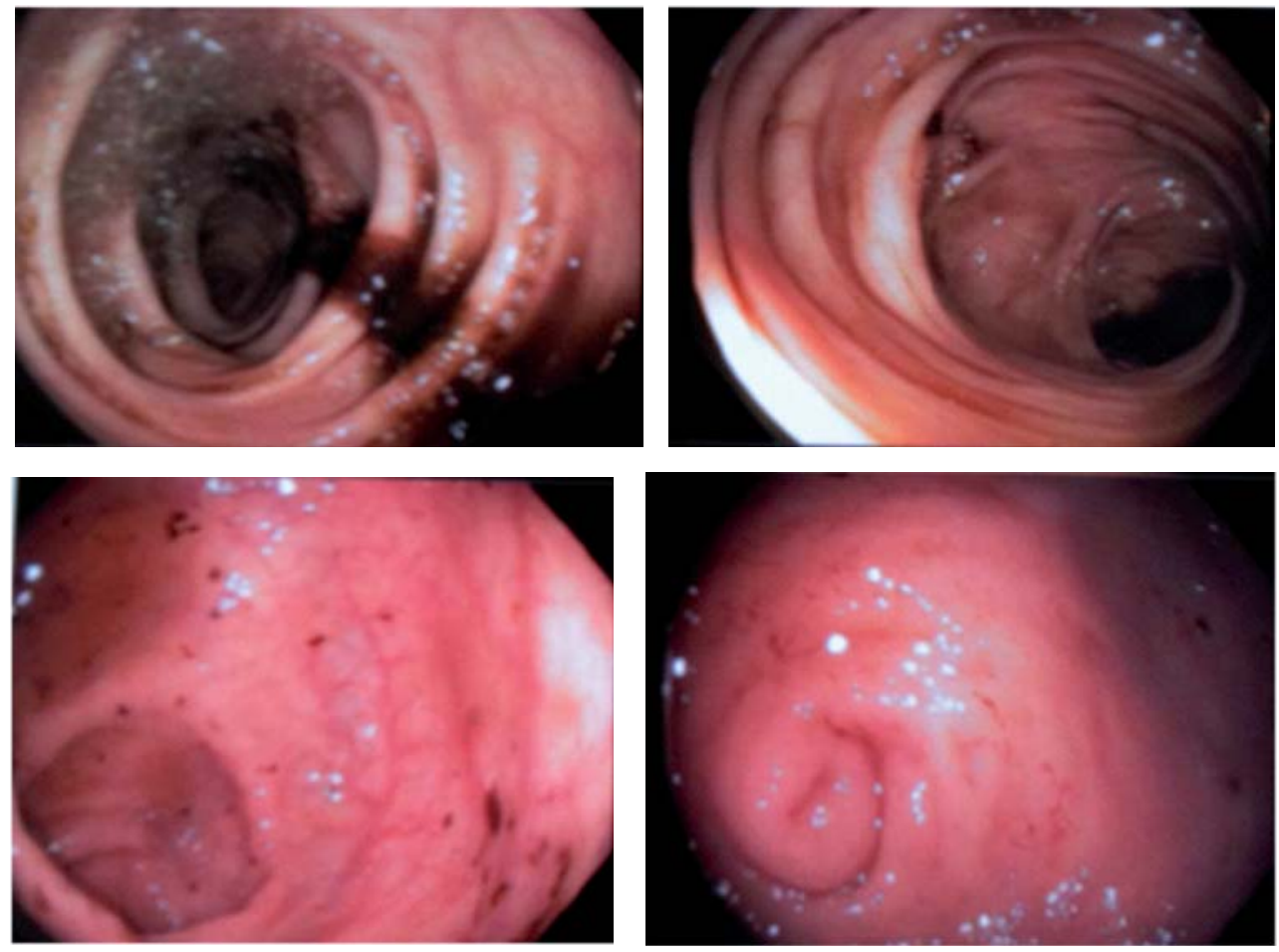

Figura 6. Colonoscopia con huellas de hemorragia sin un sitio identificable.
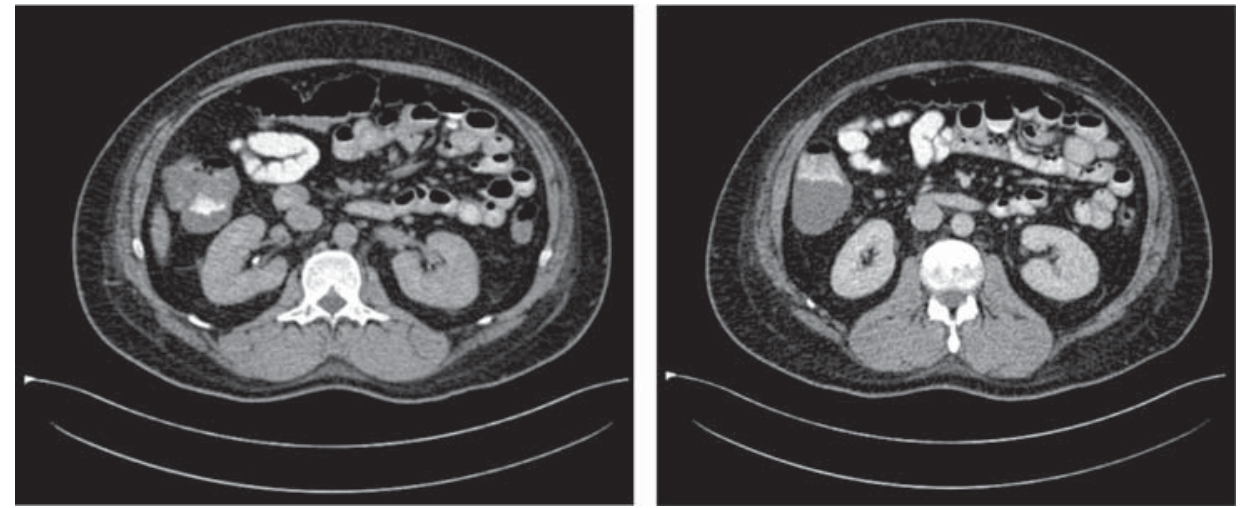

Figura 7. Tránsito intestinal con control tomográfico.

hospitalización y la realización de tránsito intestinal con control tomográfico (Figura 7). Persistió con evacuaciones de aproximadamente 2,500 $\mathrm{mL}$, con repercusión hemodinámica, requirió hemotransfusión y manejo integral del estado de choque. Fue valorado por el Servicio de Cirugía y se decidió realizar laparotomía exploradora por sangrado de tubo digestivo de etiología a determinar. En la intervención se identificaron múltiples

divertículos en el borde mesentérico del yeyuno proximal, a $40 \mathrm{~cm}$ del ángulo de Treitz (Figura 8), en un segmento de $20 \mathrm{~cm}$, por lo cual se realizó resección intestinal con anastomosis mecánica, sin otros incidentes o hallazgos transoperatorios. Se envió la pieza a Anatomía Patológica, que reportó divertículos con notable adelgazamiento de la capa muscular del yeyuno y hemorragia reciente (Figura 9). 

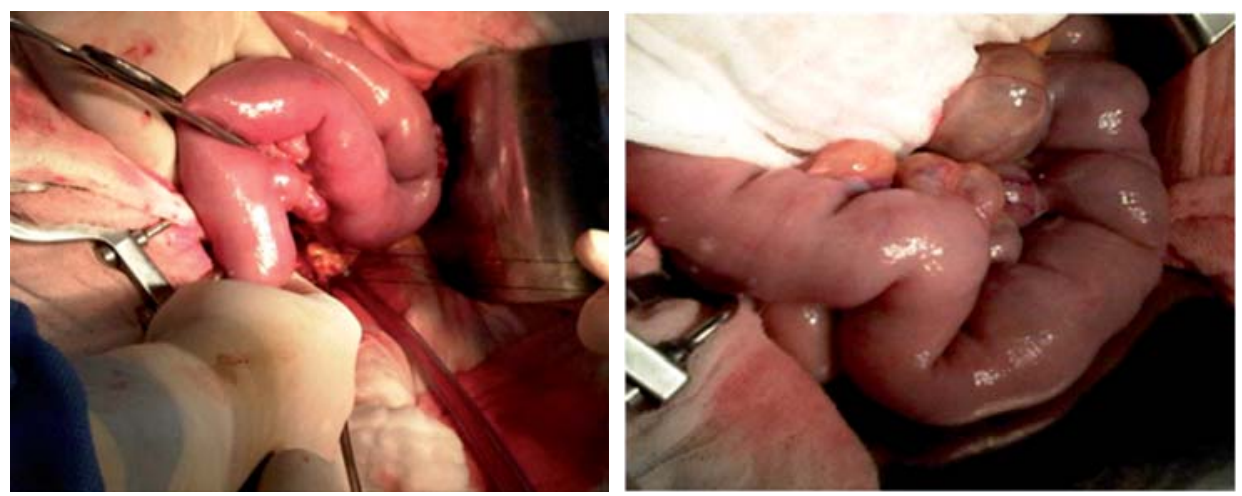

Figura 8.

LAPE con resección de yeyuno. Se realizó una enterotomía e introdujo una cánula de Yankauer en uno de los divertículos. Véase la delgadez de la pared del mismo.
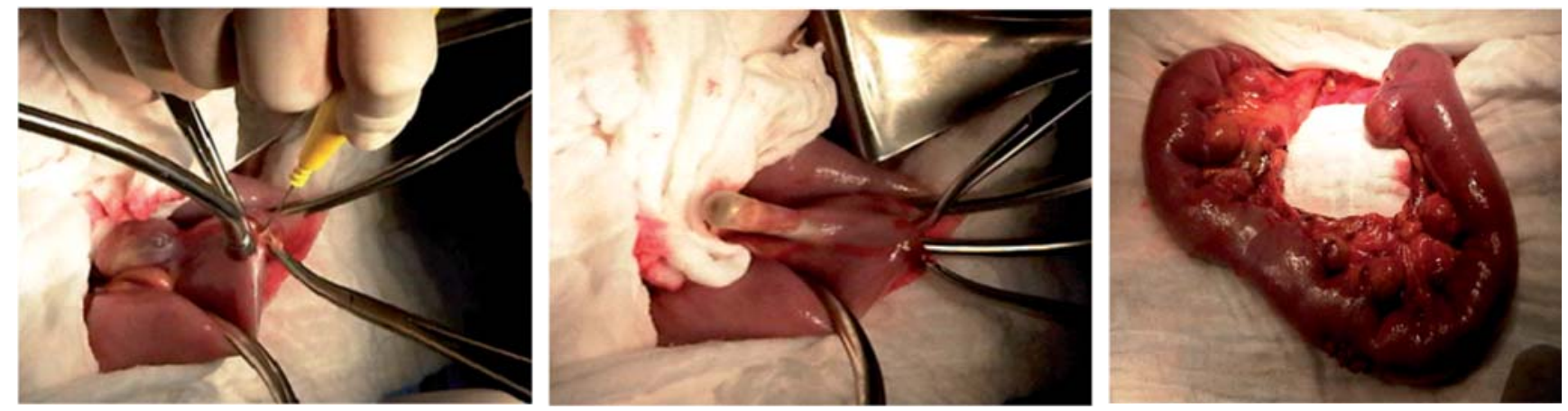

\section{DISCUSIÓN}

La hemorragia de tubo digestivo es un motivo importante de consulta en urgencias. La mayoría requiere tratamiento inmediato ante la posible inestabilidad hemodinámica e incremento de la morbimortalidad. El diagnóstico de los divertículos de duodeno y yeyuno presenta dificultad debido a que no existe una presentación característica, siendo los síntomas vagos e inespecíficos. Los divertículos duodenales son poco frecuentes y menos de $5 \%$ requiere tratamiento quirúrgico. ${ }^{2,8,9}$ Su frecuencia es incierta y varía de 0.16 a $5.76 \%$ en los hallazgos radiológicos, y llega hasta $20 \%$ en los casos sometidos a autopsia. Generalmente, se localizan a $2 \mathrm{~cm}$ de la ampolla de Vater, en la segunda porción del duodeno. El estudio de mayor sensibilidad para la detección de divertículos duodenales es la endoscopia de tubo digestivo alto, con una capacidad de detección de hasta $20 \%$. La serie de esófago-gastroduodenal resulta útil pese a la baja sensibilidad diagnóstica (de 1 a 5\%). En este caso, el estudio endoscópico fue el que permitió realizar el diagnóstico; sin embargo, la serie esófago-gastroduodenal con bario fue el estudio radiológico que aportó la información más importante para el diagnóstico definitivo. Si bien la sensibilidad es baja, consideramos que es el estudio que nos permite una mejor dimensión del problema.

El tratamiento quirúrgico en divertículos asintomáticos en adultos no está justificado, a diferencia del paciente pediátrico, donde el tratamiento de elección es quirúrgico. En el caso de los divertículos duodenales, el tratamiento de elección es la diverticulectomía simple de forma abierta; ${ }^{10,11}$ otras opciones son por vía endoscópica con pinzamiento o inyección de epinefrina. Actualmente, la embolización arterial selectiva está considerada como una opción más.

En cuanto a los divertículos de yeyuno, producen sintomatología clínica en 10 a 40\% de los casos, ${ }^{6,12-14}$ siendo, en su mayoría, inespecífica. En la literatura se han descrito pacientes con esta afección etiquetados como «síndrome de malabsorción», y cuyas manifestaciones clínicas son cuadros diarreicos frecuentes, de difícil control u origen, y cuya explicación no es más que la colonización de los divertículos. 

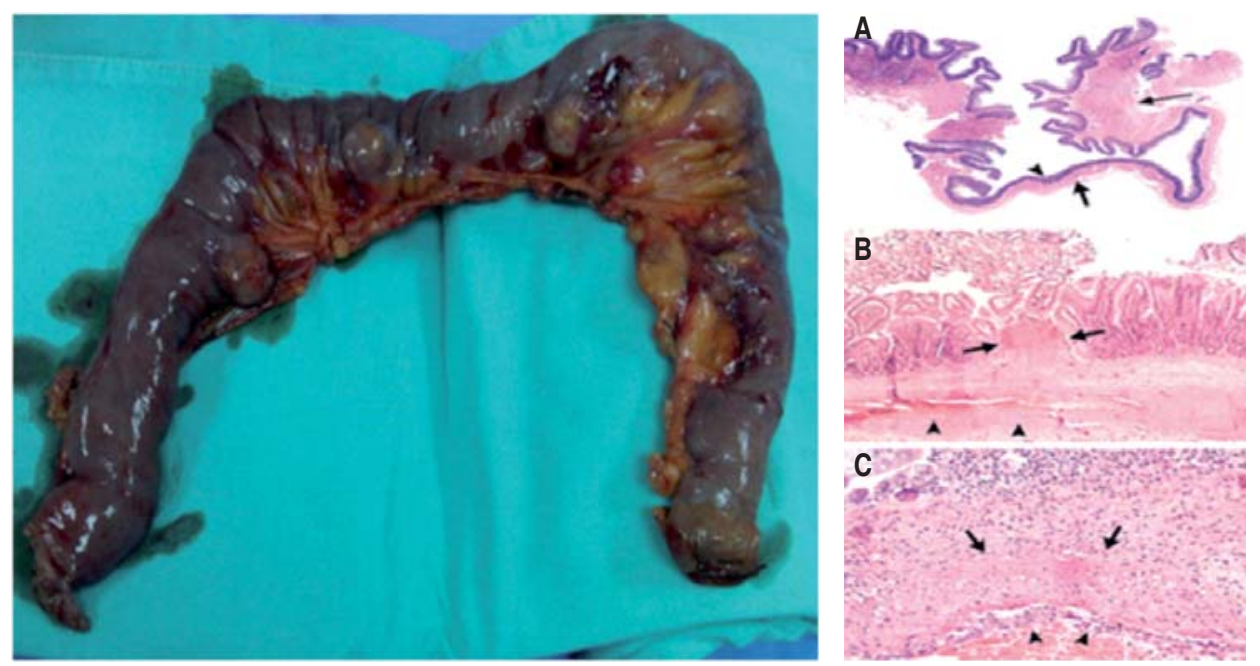

Figura 9. Pieza y corte histopatológico. Nótense los divertículos en la porción mesentérica del yeyuno.

La diverticulitis yeyunal hemorrágica es una complicación grave, y mortal en ocasiones, con frecuencia entre 2.3 y $7 \%$ de los casos. $7,12,15$ Otras complicaciones, además de la que presentó nuestro paciente, pueden ser obstrucción y perforación.

La localización más habitual de los divertículos de intestino delgado es la duodenal $(45 \%)$, seguida de la ileal, donde se localiza el divertículo de Meckel (23\%). Por estudios radiológicos baritados, se estima una prevalencia para los divertículos duodenales de $6 \%$, con una incidencia que aumenta con la edad; mientras la prevalencia de los divertículos yeyunales ronda el $0.26 \%$. Generalmente asintomáticos, su presentación clínica más habitual es en forma de dolor abdominal (49\%) y/o hemorragia digestiva $(29 \%),{ }^{8}$ esta última, presente en nuestro paciente.

El diagnóstico preoperatorio de diverticulitis yeyunal es muy poco frecuente y la mayoría de las veces se engloba en un cuadro de abdomen agudo o sangrado de tubo digestivo con etiología a determinar. La tomografía axial computarizada (TAC) puede ser útil en caso de perforación o absceso. $6,7,12$ El estudio diagnóstico de una hemorragia digestiva recurrente debe incluir una gastroscopia y, si ésta es normal, una colonoscopia. Clásicamente, cuando la gastroscopia y la colonoscopia son normales, el siguiente paso diagnóstico ha sido la realización de un estudio radiológico baritado de intestino delgado con control tomográfico. Algunos autores propugnan pasar directamente a realizar una enteroscopia alta y una ileoscopia profunda con el enteroscopio, ${ }^{1}$ cuyo rendimiento diagnóstico en el estudio de lesiones de intestino delgado oscila entre 26 y $75 \%$. El enteroscopio puede ser introducido en el intestino delgado entre 40-150 cm más allá del ligamento de Treitz; también es posible realizar esta prueba con apoyo radiológico para medir más correctamente la profundidad alcanzada y la localización de las lesiones. Recientemente se ha desarrollado una nueva técnica endoscópica que permite la visualización no invasiva de la totalidad del intestino delgado. Consiste en la ingesta de una cápsula de $30 \times 11 \mathrm{~mm}$ (Given Imaging, Ltd., Yokneam, Israel) que contiene una cámara y transmite imágenes obtenidas a su paso por el intestino durante un periodo de aproximadamente siete horas, a una frecuencia variable. ${ }^{16}$

El tratamiento clásico de la diverticulitis yeyunal complicada ha sido la laparotomía con resección quirúrgica del segmento afecto y una anastomosis primaria, $5,6,7,12$ mientras que otras alternativas como la escisión local no son recomendables debido a que aumentan de manera considerable las complicaciones. ${ }^{6,17,18}$ Algunos autores, como Novak y colaboradores, ${ }^{19}$ defienden el manejo conservador de la diverticulitis yeyunal con antibióticos y drenaje 
percutáneo con control radiológico mediante tomografía axial computarizada, aunque en un estado de gravedad como en este paciente no es considerable.

\section{CONCLUSIÓN}

En resumen, en nuestros pacientes los estudios de imagen ayudaron a localizar e identificar la magnitud del problema y a dar un adecuado tratamiento en cada uno de ellos. En el segundo paciente, la laparotomía de urgencia durante un sangrado activo permitió llegar al diagnóstico de certeza de hemorragia proveniente de divertículo yeyunal y realizar el tratamiento definitivo. Así mismo es importante tomar en cuenta esta entidad en el diagnóstico diferencial del sangrado del tubo digestivo cuyo origen es desconocido. Idealmente, el paciente deberá estar en las condiciones más óptimas posibles y previamente ser recuperado del estado de choque, teniendo todos los elementos de apoyo disponibles, como sangre y sus derivados, apoyo ventilatorio, profilaxis antibiótica, medidas antiembólicas, etcétera. Afortunadamente, al realizar la laparotomía exploradora se identificó la causa de la hemorragia; sin embargo, a pesar de ello, en ocasiones es menester realizar enterotomías e introducir de manera segmentaria un endoscopio que permita encontrarla. Presentamos estos casos por tratarse de entidades que deben ser tomadas en cuenta entre los diagnósticos diferenciales de la hemorragia digestiva de origen oscuro.

\section{CONFLICTO DE INTERESES}

Los autores declaran no tener ningún conflicto de intereses.

\section{REFERENCIAS}

1. Hayat M, Axon AT, O'Mahony S. Diagnostic yield and effect on clinical outcomes of push enteroscopy in suspected small-bowell bleeding. Endoscopy. 2000; 32: 369-372.

2. Bouhnik Y, Bitoun A, Coffin B, et al. Two way push video enteroscopy in investigation of small bowel disease. Gut. 1998; 43: 280-284.

3. Schwesinger WH, Sirinek KR, Gaskill HV, et al. Jejuno ileal causes of overt gastrointestinal bleeding: Diagno- sis, management and outcome. Am Surg. 2001; 67: 383-387.

4. Hardford WV. Divertículos de la hipofaringe y el esófago, el estómago y el intestino delgado. En: Feldman M, Scharschmidt BF, Sleisenger MH, editores. Enfermedades gastrointestinales y hepáticas. Fisiopatología, diagnóstico y tratamiento. 6a ed. Philadelphia: WB Saunders; 1998. p. 334-342.

5. Zager JS, Garbus JE, Shaw JP, et al. Jejunal diverticulosis: a rare entity with multiple presentations, a series of cases. Dig Surg. 2000; 17: 643-645.

6. Gotian A, Katz S. Jejunal diverticulitis with localized perforation and intramesentereic abscess. Am J Gastroenterol. 1998; 93: 1173-1175.

7. Azpiazu AP, de Frutos GA, Castro EE et al. Divertículos yeyunales. Dolor abdominal inespecífico. Cir Esp. 2000; 67: 214-216.

8. Chiu EJ, Shyr YM, Su CH, et al. Diverticular disease of the small bowel. Hepatogastroenterology. 2000; 47: 181-184.

9. Sharma BC, Bhasin DK, Makharia G, et al. Diagnostic value of push-type enteroscopy: a report from India. Am J Gastroenterol. 2000; 95: 137-140.

10. Yang R, Laine L. Mucosal stripping: a complication of push enteroscopy. Gastrointest Endosc. 1995; 41: 156-158.

11. Acosta N, Civac G. Long-term follow up after pushenteroscopy in patients with gastrointestinal bleeding of obscure origin (abstract). Gastrointest Endosc. 1993; 39. A264.

12. Ferri ML, Fernández E, Zarco A, Ruiz J, López JB. Abdomen agudo por divertículo yeyunal perforado. Cir Esp.1998; 63: 150-151.

13. Krishnamurthy S, Kelly MM, Rohrmann CA, et al. Jejunal diverticulosis. A heterogeneous disorder caused by a variety of abnormalities of smooth muscle or myenteric plexus. Gastroenterology. 1983; 85: 538-547.

14. Gayer G, Zissin R, Apter S, et al. Acute diverticulitis of the smallbowel: CT findings. Abdom Imaging. 1999; 24: 452-455.

15. Sibille A, Willocx R. Jejunal diverticulitis. Am J Gastroenterol. 1992; 87: 655-658.

16. Gong F, Swain P, Mills T. Wireless endoscopy. Gastrointest Endosc. 2000; 51: 725.

17. Harris LM, Volpe CM, Doerr RJ. Small bowel obstruction secondary to enterolith impaction complicating jejunal diveticulitis. Am J Gastroenterol. 1997; 92: 1538-1540.

18. Peláez MC, Rodríguez FR, Tato G, et al. Diverticulitis yeyunal perforada. Cir Esp. 2001; 69: 627-628.

19. Novak JS, Tobis J, Barkin JS. Nonsurgical management of acute jejunal diverticulitis: A review. Am J Gastroenterol. 1997; 92: 1929-1931.

Correspondencia:

Dr. José Manuel Correa Rovelo

Hospital Médica Sur.

Puente de Piedra Núm. 150, Torre 2,

Col. Toriello Guerra,

Del. Tlalpan, 14050, México, D.F.

E-mail: jcorrea@medicasur.org.mx 